\title{
METODOLOGIAS PARA AVALIAÇÃO E DIAGNÓSTICO DO ESTADO DE ISOLAMENTOS DE PAPEL IMPREGNADO COM ÓLEO MINERAL
}

\author{
Roberto Zirbes $^{* \dagger}$ \\ zirbes@eletrosul.gov.br
}

\author{
Jacqueline G Rolim* \\ jackiedlabspot.ufsc.br
}

\author{
Hans Helmut Zürn* \\ hans@labspot.ufsc.br \\ * Grupo de Sistemas de Potência -Departamento de Engenharia Elétrica \\ Universidade Federal de Santa Catarina \\ 88040-900, Florianópolis, SC \\ ${ }^{\dagger}$ ELETROSUL
}

\begin{abstract}
Cellulose impregnated with mineral oil is the most used insulating material in medium, high or extra high voltage substations, due to its efficacy and low cost. This material is employed in power transformers and its bushings, reactors, capacitors and measurement voltage and current transformers. This paper discusses the methodologies traditionally used to assess the insulating state of impregnated paper and to diagnose faults through the dissipation factor and the concentration of gases dissolved in the oil, regarding some factors that may affect those variables. Uncertainties in measured parameters and analysis methods are also considered. Some results obtained with the application of traditional methodologies (standards) for incipient fault detection based on gas analysis are compared with results obtained from the application of a artificial neural network proposed for this task.
\end{abstract}

KEYWORDS: Insulating Oil, Insulated Paper, Dissipation Factor, Dissolved Gas Analysis, Fault Diagnosis.

\section{RESUMO}

O isolamento elétrico mais comumente utilizado em subes-

Artigo submetido em 18/09/03

1a. Revisão em 27/09/05

Aceito sob recomendação do Ed. Assoc. Prof. Glauco Taranto tações de média, alta ou extra alta tensão é o de celulose impregnada com óleo mineral isolante, em função de sua eficácia e custo reduzido. Sua utilização abrange transformadores de potência e suas buchas, reatores e transformadores de medição de corrente e tensão. Este artigo discute as metodologias tradicionais utilizadas na análise de estado e no diagnóstico do isolamento a partir do fator de dissipação e da concentração de gases dissolvidos no óleo, considerando os principais fatores que afetam estes valores. São também abordados alguns aspectos que causam incerteza na medição e nas metodologias de análise. É apresentada a modelagem de uma rede neural artificial para diagnóstico e identificação de estado do isolamento a partir de dados de concentração de gases dissolvidos no óleo, sendo os resultados obtidos comparados com os das metodologias tradicionais baseadas em normas.

PALAVRAS-CHAVE: Óleo Mineral Isolante, Papel Isolante, Fator de Dissipação, Análise de Gases Dissolvidos, Diagnóstico de Faltas.

\section{INTRODUÇÃO}

A confiabilidade de um sistema de suprimento de energia elétrica tem reflexos econômicos e sociais na região suprida. Os reflexos econômicos decorrentes de uma indisponibilidade não ficam restritos ao consumidor, mas atingem também, em 
maior ou menor intensidade, aos agentes envolvidos no suprimento de energia.

Com a reestruturação do setor de energia elétrica ocorreram alterações significativas nos projetos conceituais de instalações, com o objetivo de minimizar a indisponibilidade de equipamentos. Um exemplo desta tendência é a utilização de técnicas de intervenção em equipamentos energizados, cuja aplicação, em função dos custos envolvidos, era antes restrita a determinadas situações em que a disponibilidade do fornecimento de energia era afetada.

Neste contexto, a análise dos modos de falha, as técnicas de avaliação de estado sem intervenção e de detecção de falhas incipientes assumem um papel mais amplo e relevante na filosofia de manutenção. Dentre os aspectos a serem supervisionados, a condição do isolamento de equipamentos ocupa um lugar de destaque. Historicamente a supervisão do isolamento dos transformadores de transmissão é um item relevante por suas conseqüências funcionais e pelo impacto econômico que representa uma falha nestes equipamentos.

Mais recentemente a supervisão do isolamento de transformadores de corrente passou a ser realizada de forma mais sistemática em função de uma série de falhas que resultaram na explosão de alguns dos mesmos. Nestes casos, a segurança do pessoal é o aspecto preponderante, embora o impacto econômico não seja desprezível, inclusive pela propagação dos danos a equipamentos próximos.

É crescente o emprego da monitoração contínua de parâmetros relacionados com o estado da isolação elétrica dos equipamentos, em que diversos produtos e metodologias estão sendo desenvolvidos. Como por exemplo, a medição de descargas parciais, fator de dissipação, gases dissolvidos no óleo, umidade relativa no óleo, entre outras. De uma forma geral, estes parâmetros são processados e comparados com parâmetros de referência.

\section{AVALIAÇÃO DO ESTADO DE UM ISO- LAMENTO ELÉTRICO}

A isolação elétrica em equipamentos de alta tensão tem como função primária minimizar o fluxo de corrente entre condutores submetidos a diferenciais de potencial elétrico, suportando o campo elétrico resultante. Deve, ainda, apresentar propriedades mecânicas, térmicas e químicas apropriadas ao cumprimento de sua função.

$\mathrm{Na}$ isolação de equipamentos e componentes tais como transformadores de potência, buchas, reatores e transformadores de medição é normalmente utilizado o papel impregnado com óleo isolante.

O dimensionamento dos isolamentos é feito considerando determinadas condições de trabalho, tais como faixa de temperatura de operação, máxima tensão contínua de trabalho, máxima tensão de impulso suportável, e ciclo de trabalho estimado. Os valores de referência da maioria das condições de trabalho utilizados no projeto dos isolamentos são padronizados por normas ou, em casos particulares, estabelecidos de comum acordo entre fabricantes e usuários. Para uma verificação da condição inicial do isolamento alguns ensaios de rotina e de tipo são previstos em normas específicas dos equipamentos, e outros estabelecidos de comum acordo entre fabricantes e clientes. Estes ensaios são normalmente efetuados em um ambiente de laboratório.

Os isolamentos sofrem redução de sua capacidade ao longo do tempo, mesmo quando submetidos às condições normais de projeto. Quando submetido a condições mais severas, a vida útil estimada é reduzida. Na medida em que ocorre uma redução de sua capacidade, vários processos podem ocorrer no sentido de acelerar esta redução, levando a uma incapacidade de realizar sua função, ou seja, à falha do isolamento. A recuperação de um isolamento nestas condições nem sempre é possível e os custos envolvidos são geralmente elevados. Os processos que atuam no sentido de acelerar a redução de capacidade do isolamento, quando em sua fase bem inicial, são denominadas de falhas incipientes. Estas falhas são normalmente recuperáveis com um custo reduzido.

O isolamento de papel impregnado é um isolamento composto de celulose e óleo isolante, em que cada um dos componentes possui seus processos normais de degradação e que, quando em contato, interagem entre si, podendo alterar parcialmente suas características individuais. A degradação da característica isolante da celulose e do óleo isolante envolve vários processos químicos e físicos que interagem entre si de uma forma complexa. Esta interação normalmente é no sentido de reforçar a degradação, aumentando a influência de um processo sobre o outro e conseqüentemente os reflexos nas características do isolamento.

Os principais fatores primários externos que levam à degradação da celulose e do óleo são o aquecimento, umidade e oxigênio. Alguns dos produtos da degradação, assim como a umidade e o aquecimento podem ter seus efeitos ampliados pela aplicação de um campo elétrico.

Os métodos de avaliação da degradação podem medir diretamente características intrínsecas do isolamento, produtos de sua degradação, ou ainda seus efeitos em parâmetros físicos ou químicos. Os principais métodos utilizados nas concessionárias do setor elétrico são a análise de gases dissolvidos no óleo e ensaios físico-químicos do óleo, medição de descargas parciais nos isolamentos e medições de fator de dissipação. Existe uma tendência de que estas medições sejam realizadas de forma contínua com o equipamento em operação. 
A análise dos gases dissolvidos no óleo isolante tem por objetivo a avaliação da condição de normalidade do isolamento papel-óleo, com a identificação de eventuais processos de falhas que estejam ocorrendo. Os processos identificáveis têm como origem o sobreaquecimento do isolamento e a ocorrência de descargas elétricas em seu interior, em vários níveis de intensidade. A estimativa das falhas incipientes é feita em um primeiro momento por valores de referência estabelecidos estatisticamente e posteriormente pela análise dos resultados por especialistas. Este método, que será o foco das metodologias a serem apresentadas nos itens 4 e 5, tem o mérito de poder ser utilizado com o equipamento em operação normal, seja por amostragem periódica do óleo, seja pela monitoração contínua.

A medição do fator de dissipação, normalmente é relacionada com a condição do isolamento através de valores de referenciais pré-fixados, em geral baseados em médias estatísticas. É associado ao calor dissipado nos isolamentos, sendo este algumas vezes o referencial para o estabelecimento de valores limites de operação. Este método é tradicionalmente utilizado com o equipamento fora de operação e desconectado do sistema. Nos últimos anos alguns sistemas de medição contínua com o equipamento em operação têm sido desenvolvidos comercialmente.

A medição de descargas parciais, descargas elétricas onde não ocorre a ruptura total do isolamento, tem por objetivo determinar sua ocorrência no isolamento, caracterizando assim este processo de falha. Sua medição pode ser realizada pelo sinal elétrico gerado pela descarga ou acusticamente pelo sinal gerado pela onda de choque da descarga. A medição do sinal elétrico gerado permite uma associação com a intensidade da descarga e uma caracterização da configuração dos eletrodos envolvidos. A medição dos sinais acústicos não permite uma caracterização confiável da intensidade da descarga, mas permite a localização da fonte espacialmente. O método acústico é normalmente utilizado com o equipamento em operação, enquanto que o método de medição do sinal elétrico apenas nos últimos anos é utilizado nestas condições.

\section{ACOMPANHAMENTO DO ÓLEO MINE- RAL EM SERVIÇO}

\subsection{Características Gerais}

O óleo mineral isolante é obtido a partir do refino de uma fração dos hidrocarbonetos coletados durante a destilação do petróleo cru e suas características variam conforme sua procedência e tecnologia empregadas.

Maiores detalhes sobre a mistura de compostos dos óleos isolantes e suas características podem ser encontrados em Wil- son (1980) e Rouse (1998).

Tendo em vista a presença de um elevado número de componentes em diferentes quantidades em sua composição, a caracterização de um óleo mineral para utilização como isolante não é feita pela composição da mistura do mesmo, mas por uma série de parâmetros físico-químicos que são afetados por sua composição e que apresentam reflexos em sua utilização. Assim, o estabelecimento de limites para estes parâmetros tem como objetivo alcançar uma uniformidade de comportamento como isolante e referências de qualidade para sua utilização.

As características físico-químicas, os valores limites, assim como procedimentos e metodologias para sua medição são padronizados por entidades tais como ABNT, IEC e ASTM, entre uma série de outros órgãos oficiais normativos e apresentam diferenças entre si, não somente na variável monitorada, mas principalmente nos limites estabelecidos. Como exemplo podem ser citadas algumas destas características ou ensaios, tais como aparência, densidade máxima, viscosidade, ponto de fluidez, ponto de fulgor, índice de neutralização, tensão interfacial, cor, teor de água, presença de cloretos e sulfatos, presença de enxofre corrosivo, ponto de anilina, rigidez dielétrica, fator de potência, estabilidade a oxidação, rigidez dielétrica a impulso, índice de refração e outros. Uma parte destas características do óleo é utilizada não somente como parâmetro de projeto de isolamentos, mas também para acompanhamento da adequação do óleo em uso para sua função.

Para um maior detalhamento sobre as padronizações podem ser consultadas as referências Wilson (1980) e IEC 60599 (1999).

\subsection{Umidade}

A umidade pode encontrar-se nos óleos isolantes basicamente sob três formas distintas, na forma de solução, em estado de emulsão ou em dispersão grosseira.

A umidade sob a forma de vapor é solúvel no óleo em quantidades que dependem da composição do óleo, temperatura e pressão. Ao atingir a saturação, a quantidade excedente não se encontra em solução, podendo estar sob a forma de emulsão (para partículas de dimensões inferiores a $1 \mu \mathrm{m}$ ). No estado de emulsão, como as partículas de umidade formadas possuem pequenas dimensões, não ocorre a precipitação devido às forças de tensão superficial e viscosidade do óleo. Quando as partículas formadas possuem dimensões maiores, a água se encontra em uma dispersão grosseira e ocorre sua precipitação em forma de gotículas.

A solubilidade da umidade no óleo varia em função de 
sua composição química quando novo (origem e qualidade do refino) (Koritsky,1970) e com seu estado de degradação quando em uso.

Outra maneira de expressar o teor de umidade no óleo isolante é através do conceito da solubilidade relativa, que é considerada freqüentemente como umidade relativa do óleo (Oommen, 1991),(Mamishev et al., 2001). A solubilidade relativa é dada pela relação entre o teor de umidade existente no óleo em uma determinada temperatura e o valor do teor umidade da máxima solubilidade para a mesma temperatura. A aplicação mais intensiva deste conceito vem ocorrendo nos últimos anos, em parte pela própria tecnologia de medição sendo desenvolvida, e por permitir uma melhor visualização das correlações entre variáveis envolvidas e comportamentos que envolvem a saturação, isto é a passagem da umidade para o estado de água livre na solução.

Os tempos envolvidos para o estabelecimento da condição de equilíbrio são variáveis e dependem não somente das condições da fonte de umidade, mas também de condições como temperatura e pressão do sistema a ser equilibrado.

\subsection{Degradação do Óleo Mineral Isolante}

A degradação do óleo mineral isolante abrange uma série de fenômenos, tais como reações de oxidação, reações de polimerização, condensação, rupturas de cadeias, etc..

Os fatores que mais influenciam a degradação são a presença de oxigênio, temperaturas relativamente elevadas e contato com substâncias quimicamente ativas de vários materiais que constituem o conjunto do isolamento ou equipamento.

A existência de descargas de baixa intensidade provoca a formação de gases e a polimerização do óleo, resultando na formação de borra ou sabões insolúveis.

De um modo geral, o processo de envelhecimento se inicia com a formação de radicais livres, que são hidrocarbonetos que perderam um átomo de hidrogênio pela ação catalítica dos metais presentes, especialmente o cobre. A partir dos radicais livres, são formados hidroperóxidos que são produtos instáveis e podem liberar oxigênio. Posteriormente são formados ácidos e outros produtos polares que são quimicamente ativos. Nesta fase existe um aumento de acidez e do fator de dissipação do óleo. Ocorre um aumento da tensão interfacial e um aumento da capacidade de dissolução de água. Um eventual aumento da quantidade de água dissolvida poderá ter influência na rigidez dielétrica. Em uma fase posterior, ocorre a polimerização a partir dos produtos do envelhecimento com a formação de substâncias resinosas ou borra. Estes produtos são pouco solúveis no óleo, vindo a se depositar sobre o isolamento ou em áreas de circulação do óleo.
A deposição desta borra, que atua como isolante térmico e como restrição ao fluxo do óleo, faz com que a temperatura do isolamento seja elevada. Um aumento de temperatura no óleo atua no sentido de realimentar positivamente o processo de deterioração do óleo, acelerando o mesmo.

Para temperaturas mais elevadas, o óleo mineral sofre uma decomposição, em que vários compostos são formados pelo fracionamento dos compostos de hidrocarbonetos. Os mecanismos são complexos, sendo basicamente quebras de ligações carbono-hidrogênio e carbono-carbono. Os radicais livres formados se recombinam formando novos gases. Estes processos dependem dos hidrocarbonetos que formam o óleo e da distribuição de energia e da temperatura na região de fracionamento. As reações ocorrem de forma estequiométrica; assim sem informações precisas quanto aos compostos presentes no óleo mineral e as condições de energia do ambiente da reação, o uso da cinética química não permite uma previsão razoável dos produtos de uma falha. Uma aproximação possível é considerar que todos os hidrocarbonetos presentes no óleo irão se decompor nos mesmos produtos e que estes produtos estarão em equilíbrio uns com os outros. Utilizando constantes de equilíbrio conhecidas das reações de decomposição relevantes e considerando a aproximação de que a reação ocorre em um equilíbrio térmico de temperatura constante, é possível através de um modelo termodinâmico calcular a pressão parcial de cada gás em função da temperatura. Um exemplo desta estimativa é apresentada pela IEEE C57.104 (1991), reproduzida na figura 1. Os gases considerados como subprodutos neste modelo são hidrogênio $\left(\mathrm{H}_{2}\right)$, metano $\left(\mathrm{CH}_{4}\right)$, acetileno $\left(\mathrm{C}_{2} \mathrm{H}_{2}\right)$, etileno $\left(\mathrm{C}_{2} \mathrm{H}_{4}\right)$ e etano $\left(\mathrm{C}_{2} \mathrm{H}_{6}\right)$, embora existam outros gases formados na decomposição por ação da temperatura. Podem ser formadas ainda, dependendo da temperatura envolvida, partículas sólidas de carbono e produtos de polimerização.

Desta forma é possível estimar a temperatura em que ocorreu a decomposição do óleo em função das concentrações dos gases dissolvidos no mesmo ou das relações entre suas concentrações.

Com base na estimativa da temperatura de decomposição do óleo, é possível associar uma provável falha que possa estar ocorrendo no mesmo, por exemplo, em uma falha de origem elétrica, onde podemos encontrar descargas de baixa intensidade, como descargas parciais ou arcos intermitentes de baixa intensidade ou descargas de alta intensidade, como arcos elétricos.

À medida que a intensidade de uma descarga elétrica atinge proporções de uma descarga contínua ou a condição de um arco elétrico, a temperatura do óleo na região em torno desta descarga atinge temperaturas que vão de $700{ }^{\circ} \mathrm{C}$ a valores da ordem de $1800^{\circ} \mathrm{C}$. Assim, quando ocorrem descargas de 


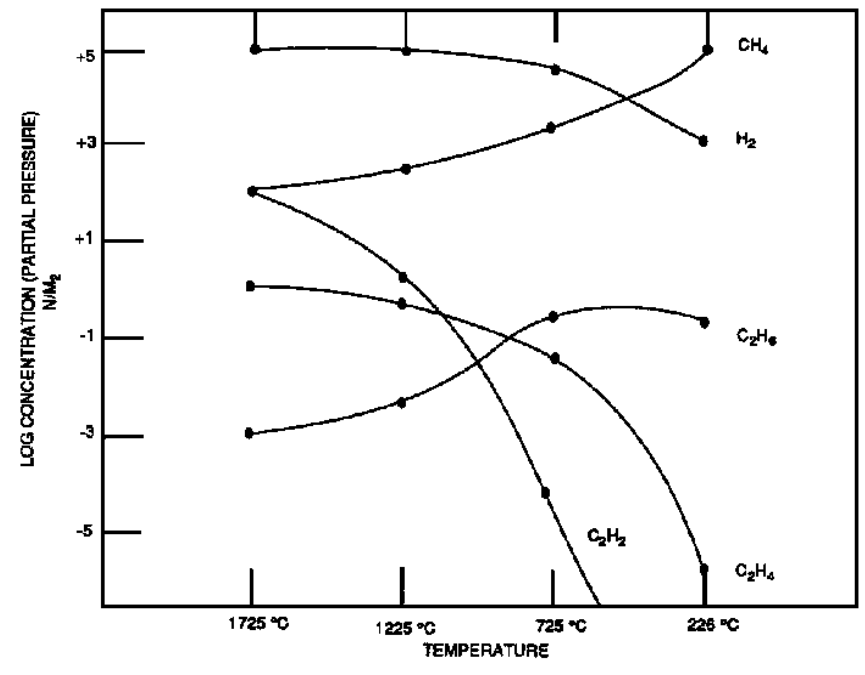

Figura 1: Pressões parciais dos gases em equilíbrio térmico em função da temperatura

maior intensidade, portanto ocasionando regiões com temperaturas elevadas, a concentração de acetileno se torna significativa. Quando a descarga é de baixa intensidade o mesmo não é detectado ou apenas traços de sua presença. Desta forma a descarga elétrica de alta intensidade é associada à presença do acetileno. Sua presença em um nível significativo no óleo é uma evidência de que em algum momento ocorreu uma descarga. Logo, se entre duas verificações ocorrer uma alteração significativa da concentração deste gás é uma evidência de que ocorreu uma descarga.

Outro exemplo é o uso das relações entre os gases. Para temperaturas entre 150 e $400^{\circ} \mathrm{C}$ os gases mais significativos são os de baixo peso molecular como o hidrogênio e o metano, com concentrações mais elevadas de metano. Um aumento, mesmo que relativamente pequeno de temperatura, faz com que a concentração de hidrogênio seja superior à do metano. Assim a relação entre os dois gases é totalmente alterada. $\mathrm{O}$ valor desta relação é uma evidência para discriminar entre falhas térmicas de baixa intensidade.

Estes exemplos embora simples e parciais ilustram os mecanismos básicos da metodologia de diagnóstico de falhas através dos gases dissolvidos.

Quando a formação de gases acontece em um intervalo de tempo reduzido e com uma pressão superior à do óleo na região, bolhas podem ser formadas. Os gases destas bolhas são geralmente dissolvidos no óleo, caso o tempo de permanência de contato com o mesmo seja prolongada. Quando o sistema é fechado, normalmente após um intervalo de tempo ocorre a dissolução dos gases no óleo, caso o óleo não esteja em seu estado de saturação. Quando a quantidade de gases dissolvidos é elevada e ocorrer uma alteração na tem- peratura que modifique o valor da solubilidade de saturação, os gases antes dissolvidos podem ser liberados na forma de bolhas. A existência de bolhas de gases não dissolvidos altera a condição de rigidez dielétrica, levando a uma ruptura do isolamento.

\subsection{Alguns Fatores que Geram Incertezas na Análise de Gases}

Vários fatores intervêm na incerteza tanto nos valores medidos de teor de gases dissolvidos, quanto na interpretação dos mesmos.

Apenas parte dos produtos de degradação do óleo por ação da temperatura é utilizada nos métodos normalmente empregados para a análise (Galland et al., 1972). Dentre estes gases, alguns são formados em uma ampla faixa de temperatura em quantidades variáveis. Além disso, outros processos físicos e químicos podem produzir estes gases, como por exemplo, a formação de hidrogênio. Pela baixa energia de ativação necessária à sua formação ele é produzido em uma ampla faixa de temperatura. Reações de hidrólise que ocorrem no interior dos equipamentos por ação de metais, também produzem este gás (Pugh, 1973). Alguns tipos de metais utilizados na constituição de equipamentos possuem em seu interior hidrogênio em função de seu processo de fabricação (Sokolov et al.,2001).

Além dos mecanismos de solubilidade dos gases envolvendo as condições de equilíbrio entre as fases do gás e do óleo, existe a migração de gases entre a celulose e o óleo. Este fenômeno depende da temperatura do meio e pode levar à determinação de concentrações de gases bastante diferentes (Kan e Miyamoto, 1995). Um exemplo desta variação é apresentado na Tabela 1 (Sokolov et al.,2001), onde para um mesmo transformador é feita a amostragem dos gases dissolvidos no óleo em duas temperaturas diferentes.

Este comportamento não é perfeitamente quantificado, dependendo inclusive da relação volume de papel e volume de óleo, podendo levar a diferentes interpretações em função do aspecto a ser analisado, no entanto o uso de um referencial de temperatura pode limitar a incerteza envolvida.

Os processos de falha, embora estudados separadamente, não ocorrem necessariamente de forma isolada. Por exemplo, a ocorrência de descargas parciais pode causar a polimerização do óleo com a conseqüente deposição deste produto sobre o isolamento. Esta deposição, dependendo da localização e da intensidade, pode ocasionar sobreaquecimento em função da alteração de troca de calor.

A distribuição da temperatura nos equipamentos não é uniforme, alguns deles sofrem uma influência significativa de 
Tabela 1: Efeito da temperatura na distribuição de gases no óleo

\begin{tabular}{|c|c|c|}
\hline $\begin{array}{c}\text { Tipos de } \\
\text { gases }\end{array}$ & $\begin{array}{c}\text { Concentração } \\
\text { a } 20{ }^{\circ} \mathrm{C}\end{array}$ & $\begin{array}{c}\text { Concentração } \\
\text { a } 64{ }^{\circ} \mathrm{C}\end{array}$ \\
\hline $\mathrm{H}_{2}(\mathrm{ppm})$ & Traços & 56 \\
\hline $\mathrm{CH}_{4}(\mathrm{ppm})$ & 172 & 269 \\
\hline $\mathrm{C}_{2} \mathrm{H}_{4}(\mathrm{ppm})$ & 78 & 147 \\
\hline $\mathrm{C}_{2} \mathrm{H}_{2}(\mathrm{ppm})$ & - & 1.3 \\
\hline $\mathrm{C}_{2} \mathrm{H}_{6}(\mathrm{ppm})$ & 56 & 90 \\
\hline $\mathrm{CO}(\mathrm{ppm})$ & 923 & 1163 \\
\hline $\mathrm{CO}_{2}(\mathrm{ppm})$ & 1929 & 2654 \\
\hline $\mathrm{O}_{2}(\%)$ & 0.08 & 0.09 \\
\hline $\mathrm{N}_{2}(\%)$ & 2.9 & 5.5 \\
\hline
\end{tabular}

uma variação de carga, enquanto para outros não existe uma correlação entre a energia dissipada e a formação de gases. A própria localização da falha e sua intensidade e tempo de duração são aspectos importantes na geração de gases. Pontos próximos à falha tendem a apresentar concentrações de gases mais elevadas (Sokolov et. al, 1999).

O tipo de equipamento e seu projeto têm influência no volume de gases gerados (Duval e de Pablo, 2001). Tradicionalmente a análise de gases é utilizada em transformadores de potência. Entretanto, é uma técnica recomendada para equipamentos que utilizam isolamento de papel impregnado imersos em óleo. A recomendação da IEC 60599 para análise de gases dissolvidos reconhece um comportamento diferenciado entre os equipamentos quanto à geração de gases, estabelecendo diferentes limites por tipo de equipamento.

Os métodos e procedimentos de medição também contribuem para a incerteza da determinação da concentração de gases dissolvidos. Para ilustrar esta incerteza são apresentados na Tabela 2 alguns resultados de uma comparação interlaboratorial feita pela IEC (Duval, 1989).

Não são apresentados neste trabalho os aspectos envolvendo as características de incerteza de cada método de medição. Entretanto é importante salientar que para baixas concentrações de gases a incerteza é significativa, e no cálculo das relações entre os gases pode representar uma variação de até $40 \%$. Este fato é particularmente importante para transformadores de medição que apresentam normalmente baixas concentrações de gases.

\section{MÉTODOS TRADICIONAIS DE SOLU- ÇÃO}

Alguns métodos utilizam como critério de diagnóstico apenas relações de concentração de gases dissolvidos, embora
Tabela 2: Resultados de comparação interlaboratorial da IEC para análise de gases

\begin{tabular}{|c|c|c|}
\hline & \multicolumn{2}{|c|}{$\begin{array}{c}\text { Desvio do valor real para } \\
\text { amostras de gás }(\%)\end{array}$} \\
\hline $\begin{array}{c}\text { Resultados dos } \\
\text { Laboratórios }\end{array}$ & $\begin{array}{c}\text { Médias } \\
\text { concentrações }\end{array}$ & $\begin{array}{c}\text { Baixas } \\
\text { concentrações }\end{array}$ \\
\hline Melhor caso & 7 & 14 \\
\hline Pior caso & 39 & 70 \\
\hline $\begin{array}{c}\text { Maior desvio de } \\
\text { um gás }\end{array}$ & 150 & 400 \\
\hline \\
1
\end{tabular} Hidrocarbonetos $(9-60 \mathrm{ppm}), \mathrm{CO}, \mathrm{CO}_{2}(100-500 \mathrm{ppm})$
2 Hidrocarbonetos $(1-10 \mathrm{ppm}), \mathrm{CO}, \mathrm{CO}_{2}(30-100 \mathrm{ppm})$

possam utilizar as concentrações individuais para validação de sua utilização.

O método de Doernenburg (Doernenburg e Gerber, 1967) utiliza duas relações principais de gases, duas relações auxiliares e seis gases. Não estabelece critérios para caracterizar se o isolamento está em condições normais, mas estabelece as condições de validade para a aplicação do método de diagnóstico em função da concentração dos gases componentes das relações.

A Tabela 3 (Bozzini et. al, 1975)apresenta os dados para análise em função das relações de gases e o diagnóstico previsto.

A Tabela 4 apresenta os dados de concentração individual dos gases para verificação da validade de aplicação do método.

O critério de validade para a utilização das relações é de que um dos gases das relações principais tenha uma concentração

Tabela 3: Critério de identificação de falha de Doernenburg

\begin{tabular}{|c|c|c|c|c|}
\hline $\begin{array}{c}\text { Relações } \\
\text { entre } \\
\text { concentrações } \\
\text { de gases }\end{array}$ & \multicolumn{2}{|c|}{$\begin{array}{c}\text { Relações } \\
\text { principais }\end{array}$} & \multicolumn{2}{c|}{$\begin{array}{c}\text { Relações } \\
\text { auxiliares }\end{array}$} \\
\hline Tipo de falha & $\mathrm{CH}_{4} / \mathrm{H}_{2}$ & $\begin{array}{c}\mathrm{C}_{2} \mathrm{H}_{2} / \\
\mathrm{C}_{2} \mathrm{H}_{4}\end{array}$ & $\begin{array}{c}\mathrm{C}_{2} \mathrm{H}_{6} / \\
\mathrm{C}_{2} \mathrm{H}_{2}\end{array}$ & $\begin{array}{l}\mathrm{C}_{2} \mathrm{H}_{2} / \\
\mathrm{CH}_{4}\end{array}$ \\
\hline $\begin{array}{c}\text { Ponto } \\
\text { quente }\end{array}$ & $>1$ & $<0.7$ & $>0.4$ & $<0.3$ \\
\hline $\begin{array}{c}\text { Descarga } \\
\text { parcial }\end{array}$ & $<0.1$ & $\begin{array}{c}\mathrm{Não}^{2} \\
\text { significativo }\end{array}$ & $>0.4$ & $<0.3$ \\
\hline $\begin{array}{c}\text { Outros } \\
\text { tipos de } \\
\text { descarga }\end{array}$ & $>1 \mathrm{e}$ & $>0.7$ & $<0.4$ & $>0.3$ \\
\hline
\end{tabular}


Tabela 4: Tabela de concentração de gases para validação do método de Doernenburg

\begin{tabular}{|c|c|c|c|c|c|}
\hline Tipo de gás & $\mathrm{H}_{2}$ & $\mathrm{CH}_{4}$ & $\mathrm{C}_{2} \mathrm{H}_{6}$ & $\mathrm{C}_{2} \mathrm{H}_{4}$ & $\mathrm{C}_{2} \mathrm{H}_{2}$ \\
\hline $\begin{array}{c}\text { Concentração } \\
\text { ppm (v/v) }\end{array}$ & 200 & 50 & 15 & 60 & 15 \\
\hline
\end{tabular}

superior ao dobro do valor na tabela 4 (Bozzini et. al., 1975) e que para as demais relações enos um dos gases tenha uma concentração superior ao constante na mesma tabela.

A IEEE C57.104 (1991) recomenda a utilização do método de Doernenburg alterando entretanto os valores de concentração de gases de referência utilizados para a validação da aplicação do método. Esta alteração corresponde aos valores de referência recomendados para uma avaliação geral quanto à condição de normalidade dos equipamentos. Como o critério para a validade de aplicação do método de Doernenburg implica que a concentração de um dos gases das relações principais seja superior ao dobro do valor previsto como referência, quando considerada a condição de aplicação da metodologia como correspondente à condição de normalidade, se admite que qualquer um dos quatro gases possa atingir valores bem mais elevados. Os valores de referência são apresentados na Tabela 5 .

Já o método de Rogers utiliza a mesma filosofia de diagnós-

Tabela 5: Tabela de concentração de gases para validação do método de Doernenburg segundo a IEEE

\begin{tabular}{|c|c|c|c|c|c|}
\hline Tipo de gás & $\mathrm{H}_{2}$ & $\mathrm{CH}_{4}$ & $\mathrm{C}_{2} \mathrm{H}_{6}$ & $\mathrm{C}_{2} \mathrm{H}_{4}$ & $\mathrm{C}_{2} \mathrm{H}_{2}$ \\
\hline $\begin{array}{c}\text { Concentração } \\
\text { ppm (v/v) }\end{array}$ & 100 & 120 & 65 & 50 & 35 \\
\hline
\end{tabular}

Tabela 6: Tabela de definição dos códigos do método de Rogers

\begin{tabular}{|c|c|c|}
\hline Relação de gases & Faixa de variação & Código \\
\hline & $<=0.1$ & 5 \\
\hline $\mathrm{CH}_{4} / \mathrm{H}_{2}$ & $>0.1,<1$ & 0 \\
\hline & $>=1,<3$ & 1 \\
\hline & $>=3$ & 2 \\
\hline $\mathrm{C}_{2} \mathrm{H}_{6} / \mathrm{CH}_{4}$ & $<1$ & 0 \\
\hline & $>=1$ & 1 \\
\hline & $<1$ & 0 \\
\hline $\mathrm{C}_{2} \mathrm{H}_{4} / \mathrm{C}_{2} \mathrm{H}_{6}$ & $>=1,<3$ & 1 \\
\hline & $>=3$ & 2 \\
\hline & $<0.5$ & 0 \\
\hline $\mathrm{C}_{2} \mathrm{H}_{2} / \mathrm{C}_{2} \mathrm{H}_{4}$ & $>=0.5,<3$ & 1 \\
\hline & $>=3$ & 2 \\
\hline
\end{tabular}

tico baseado em relações de concentrações de gases e limites de variação para estas relações. Este método sofreu várias alterações ao longo do tempo em função de adaptações realizadas para ajustar a correlação do método com resultados obtidos em equipamentos. Assim, embora os critérios originais fossem baseados em modelos teóricos aproximados, foram ao longo do tempo sendo adaptados à correlação de resultados de investigações de falha analisadas. Por estas características, este é um dos métodos mais freqüentemente utilizados e aparece com variações nos limites empregados e no número de relações de concentração de gases.

Os primeiros trabalhos utilizavam quatro relações de gases; nas Tabelas 6 e 7 é apresentado o método utilizando quatro relações e cinco gases (Rogers, 1975). A forma de apresentação do método é dividida em duas tabelas, uma contendo os valores das faixas de variação das relações e outra contendo os diagnósticos baseados no código estabelecido a partir da tabela dos valores limites das relações de gases.

Algumas empresas do Setor Elétrico ainda utilizam esta ver-

Tabela 7: Tabela de diagnóstico do método de Rogers em função do código

\begin{tabular}{|c|c|c|c|c|}
\hline $\begin{array}{l}\mathrm{CH}_{4} / \\
\mathrm{H}_{2}\end{array}$ & $\begin{array}{l}\mathrm{C}_{2} \mathrm{H}_{6} / \\
\mathrm{CH}_{4}\end{array}$ & $\begin{array}{l}\mathrm{C}_{2} \mathrm{H}_{4} / \\
\mathrm{C}_{2} \mathrm{H}_{6}\end{array}$ & $\begin{array}{l}\mathrm{C}_{2} \mathrm{H}_{2} / \\
\mathrm{C}_{2} \mathrm{H}_{4}\end{array}$ & Diagnóstico \\
\hline 0 & 0 & 0 & 0 & Deterioração normal \\
\hline 5 & 0 & 0 & 0 & Descargas parciais \\
\hline $1 / 2$ & 0 & 0 & 0 & $\begin{array}{l}\text { Sobreaquecimento - } \\
\text { abaixo de } 150^{\circ} \mathrm{C}\end{array}$ \\
\hline $1 / 2$ & 1 & 0 & 0 & $\begin{array}{l}\text { Sobreaquecimento - } \\
\text { de } 150-200^{\circ} \mathrm{C}\end{array}$ \\
\hline 0 & 1 & 0 & 0 & $\begin{array}{l}\text { Sobreaquecimento - } \\
\text { de } 200-300{ }^{\circ} \mathrm{C}\end{array}$ \\
\hline 0 & 0 & 1 & 0 & $\begin{array}{l}\text { Sobreaquecimento } \\
\text { de condutores }\end{array}$ \\
\hline 1 & 0 & 1 & 0 & $\begin{array}{l}\text { Correntes de circula- } \\
\text { ção nos enrolamen- } \\
\text { tos }\end{array}$ \\
\hline 1 & 0 & 2 & 0 & $\begin{array}{l}\text { Correntes de circu- } \\
\text { lação no núcleo e } \\
\text { tanque, sobreaqueci- } \\
\text { mento em conexões }\end{array}$ \\
\hline 0 & 0 & 0 & 1 & $\begin{array}{l}\text { Descarga descontí- } \\
\text { nua }\end{array}$ \\
\hline 0 & 0 & $1 / 2$ & $1 / 2$ & $\begin{array}{l}\text { Arco com alta ener- } \\
\text { gia }\end{array}$ \\
\hline 0 & 0 & 2 & 2 & $\begin{array}{l}\text { Descarga contínua de } \\
\text { baixa potência }\end{array}$ \\
\hline 5 & 0 & 0 & $1 / 2$ & $\begin{array}{l}\text { Descarga parcial en- } \\
\text { volvendo o papel }\end{array}$ \\
\hline
\end{tabular}


são de diagnóstico de Rogers no que se refere aos limites, embora com um número menor de diagnósticos correlacionados, em geral pela agregação de alguns diagnósticos como, por exemplo, os níveis de sobreaquecimento. A exemplo das demais versões do método, o mesmo é utilizado na avaliação de transformadores e reatores.

A revisão da NBR 7274 (1999) sugere a utilização do método de Rogers com um formato similar ao acima exposto, utilizando uma revisão em que o valor do limite mínimo da relação $\mathrm{C}_{2} \mathrm{H}_{2} / \mathrm{C}_{2} \mathrm{H}_{4}$ é alterado para 0.1 ao invés de 0.5 . A interpretação do código de diagnóstico também apresenta algumas alterações, embora basicamente apresente uma caracterização semelhante.

A norma IEEE C57.104 (1991) sugere a utilização do método de Rogers com três relações de concentração de gases e cinco gases. Este critério elimina a relação $\mathrm{C}_{2} \mathrm{H}_{6} / \mathrm{CH}_{4}$ que era utilizada para identificação de sobreaquecimento de baixa temperatura. O número de diagnósticos correlacionados com os limites é reduzido em relação às versões anteriores, conforme pode ser visto na tabela 8 .

Algumas publicações recomendam que o critério de Rogers não seja utilizado para determinação da condição de normalidade do equipamento. Que seja apenas utilizado para o diagnóstico de uma falha em função de sua baixa eficiência na

Tabela 8: Tabela de diagnóstico de Rogers sugerida pela IEEE C57-104

\begin{tabular}{|c|c|c|c|l|}
\hline Casos & $\begin{array}{c}\mathrm{C}_{2} \mathrm{H}_{2} / \\
\mathrm{C}_{2} \mathrm{H}_{4}\end{array}$ & $\begin{array}{c}\mathrm{CH}_{4} / \\
\mathrm{H}_{2}\end{array}$ & $\begin{array}{c}\mathrm{C}_{2} \mathrm{H}_{4} / \\
\mathrm{C}_{2} \mathrm{H}_{6}\end{array}$ & $\begin{array}{c}\text { Diagnóstico de falha } \\
\text { sugerido }\end{array}$ \\
\hline 0 & $<0.1$ & $\begin{array}{c}>0.1 \\
\mathrm{e} \\
<1.0\end{array}$ & $<1.0$ & Condições normais \\
\hline 1 & $<0.1$ & $<0.1$ & $<1.0$ & $\begin{array}{l}\text { Arco de baixa ener- } \\
\text { gia / descarga parcial }\end{array}$ \\
\hline 2 & $\begin{array}{c}0.1 \mathrm{a} \\
3.0\end{array}$ & $\begin{array}{c}0.1 \mathrm{a} \\
1.0\end{array}$ & $>3.0$ & $\begin{array}{l}\text { Arco - Descarga de } \\
\text { alta energia }\end{array}$ \\
\hline 3 & $<0.1$ & $\begin{array}{c}>0.1 \\
\mathrm{e} \\
<1.0\end{array}$ & $\begin{array}{l}1.0 \mathrm{a} \\
3.0\end{array}$ & $\begin{array}{l}\text { Sobreaquecimento - } \\
\text { baixas temperaturas }\end{array}$ \\
\hline 4 & $<0.1$ & $>0.1$ & $\begin{array}{l}1.0 \mathrm{a} \\
\text { a }\end{array}$ & $\begin{array}{l}\text { Sobreaquecimento } \\
\text { temperatura < 700 } \\
{ }^{\circ} \mathrm{C}\end{array}$ \\
\hline 5 & $<0.1$ & $>0.1$ & $>3.0$ & $\begin{array}{l}\text { Sobreaquecimento } \\
\text { temperatura > 700 } \\
{ }^{o} \mathrm{C}\end{array}$ \\
\hline
\end{tabular}

caracterização da condição de normalidade.

A revisão da NBR 7274 é baseada na IEC 599 de 1978 e sugere a utilização do método apresentado na Tabela 9.

É possível observar que as faixas de variação das relações apresentam superposições, não permitindo uma interpretação única. Também em muitos casos não existe um diagnóstico definido, pois as combinações de variações que podem ocorrer nas relações são maiores que aquelas definidas na tabela do método. Este fato ocorre com todas as variantes dos métodos.

A IEC 60599 apresenta um método de diagnóstico semelhante aos anteriormente apresentados. A revisão de 1999 apresenta as mesmas relações básicas de concentrações com uma alteração dos limites e principalmente dos casos característicos de falha. Como pode ser observado na tabela 10 , para a definição do estado de normalidade não são utilizadas as relações de concentração.

A identificação da condição de normalidade passa a ser estimada pela concentração de gases. A sugestão de limites de normalidade encontrada na literatura é extremamente variável pela sua dependência de características dos dados utilizados e a incerteza associada aos mesmos, conforme citado anteriormente. O nível de sobreposição de diagnósticos aumenta, por exemplo, na identificação de falhas de origem elé-

Tabela 9: Tabela para interpretação da análise de gases de transformadores e reatores

\begin{tabular}{|c|c|c|c|}
\hline $\begin{array}{c}\mathrm{C}_{2} \mathrm{H}_{2} / \\
\mathrm{C}_{2} \mathrm{H}_{4}\end{array}$ & $\begin{array}{c}\mathrm{CH}_{4} / \\
\mathrm{H}_{2}\end{array}$ & $\begin{array}{c}\mathrm{C}_{2} \mathrm{H}_{4} / \\
\mathrm{C}_{2} \mathrm{H}_{6}\end{array}$ & Diagnóstico \\
\hline$<0.1$ & 0.1 a 1.0 & $<1.0$ & $\begin{array}{c}\text { Envelhecimento } \\
\text { normal }\end{array}$ \\
\hline$<0.1$ & $<0.1$ & $<1.0$ & $\begin{array}{c}\text { Descarga parcial } \\
\text { baixa energia }\end{array}$ \\
\hline $0.1 \mathrm{a} 3.0$ & $<0.1$ & $<1.0$ & $\begin{array}{c}\text { Descarga parcial } \\
\text { alta energia }\end{array}$ \\
\hline$>0.1$ & $0.1 \mathrm{a} 1.0$ & $>1.0$ & $\begin{array}{c}\text { Arco - descarga } \\
\text { baixa energia }\end{array}$ \\
\hline $0.1 \mathrm{a} 3.0$ & $0.1 \mathrm{a} 1.0$ & $>3.0$ & $\begin{array}{c}\text { Arco - descarga } \\
\text { alta energia }\end{array}$ \\
\hline$<0.1$ & $>1.0$ & $<1.0$ & $\begin{array}{c}\text { Sobreaquecimento } \\
150^{\circ} \mathrm{C}<\mathrm{t}<300^{\circ} \mathrm{C}\end{array}$ \\
\hline$<0.1$ & $>1.0$ & $1.0 \mathrm{a} 3.0$ & $\begin{array}{c}\text { Sobreaquecimento } \\
30{ }^{\circ} \mathrm{C}<\mathrm{t}<700^{\circ} \mathrm{C}\end{array}$ \\
\hline$<0.1$ & $>1.0$ & $>3.0$ & $\begin{array}{c}\text { Sobreaquecimento } \\
\mathrm{t}>700^{\circ} \mathrm{C}\end{array}$ \\
\hline
\end{tabular}


Tabela 10: Tabela para interpretação da análise de gases de transformadores e reatores segundo a IEC 60599.

\begin{tabular}{|c|c|c|c|c|}
\hline Caso & $\begin{array}{c}\text { Falha } \\
\text { característica }\end{array}$ & $\begin{array}{l}\mathrm{C}_{2} \mathrm{H}_{2} / \\
\mathrm{C}_{2} \mathrm{H}_{4}\end{array}$ & $\begin{array}{c}\mathrm{CH}_{4} / \\
\mathrm{H}_{2}\end{array}$ & $\begin{array}{l}\mathrm{C}_{2} \mathrm{H}_{4} / \\
\mathrm{C}_{2} \mathrm{H}_{6}\end{array}$ \\
\hline PD & $\begin{array}{l}\text { Descarga } \\
\text { Parcial }\end{array}$ & $\begin{array}{l}\text { Não } \\
\text { signifi- } \\
\text { cativo }\end{array}$ & $<0.1$ & $<0.2$ \\
\hline D1 & $\begin{array}{c}\text { Descarga de } \\
\text { baixa energia }\end{array}$ & $>1$ & $\begin{array}{c}0.1 \mathrm{a} \\
0.5\end{array}$ & $>1$ \\
\hline D2 & $\begin{array}{l}\text { Descarga de } \\
\text { alta energia }\end{array}$ & $\begin{array}{c}0.6 \mathrm{a} \\
2.5\end{array}$ & $\begin{array}{c}0.1 \mathrm{a} \\
1.0\end{array}$ & $>2$ \\
\hline T1 & $\begin{array}{c}\text { Sobre- } \\
\text { aquecimento } \\
\mathrm{t}<300^{\circ} \mathrm{C}\end{array}$ & $\begin{array}{l}\text { Não } \\
\text { signifi- } \\
\text { cativo }\end{array}$ & $\begin{array}{c}>1, \\
\text { mas } \\
\text { Não } \\
\text { signifi- } \\
\text { cativo }\end{array}$ & $<1$ \\
\hline $\mathrm{T} 2$ & $\begin{array}{c}\text { Sobre- } \\
\text { aquecimento } \\
300{ }^{\circ} \mathrm{C}<\mathrm{t}< \\
700{ }^{\circ} \mathrm{C}\end{array}$ & $<0.1$ & $>1$ & $1 \mathrm{a} 4$ \\
\hline T3 & $\begin{array}{c}\text { Sobre- } \\
\text { aquecimento } \\
\mathrm{t}>700^{\circ} \mathrm{C}\end{array}$ & $<0.2$ & $>1$ & $>4$ \\
\hline
\end{tabular}

trica (D1,D2), como pode ser visto na tabela 10, mas é esperado um percentual de casos sem um diagnóstico definido menor que a versão anterior.

Para o estudo de algumas técnicas de classificação ou de diagnóstico serão utilizados dois conjuntos de dados de análises de gases dissolvidos no óleo. Para cada conjunto de gases medidos a condição dos equipamentos foi determinada através de medições específicas e inspeções visuais feitas por especialistas.

Um dos conjuntos de dados é parte da base de dados da IEC TC 10 (Duval e de Pablo, 2001). Esta publicação de base de dados apresenta vários conjuntos de dados relativos a diferentes equipamentos, sendo organizada por tipo de falha e identificada por tipo de equipamento. Fica claro que os comportamentos dos vários equipamentos são diferenciados, não sendo conveniente uma tentativa de análise considerando em um mesmo conjunto de falhas todos os equipamentos indistintamente. Assim o conjunto escolhido, para efeito desta aplicação, como representativo dos transformadores é o conjunto relativo a transformadores sem conexão com comuta- dores sob carga. A este conjunto foram acrescidos os dois limites previstos na IEC para a caracterização da condição de normalidade dos transformadores.

O outro conjunto de dados utilizado é parte de um banco de dados do CEPEL; neste conjunto constam transformadores de vários níveis de tensão, desde $13.8 \mathrm{kV}$ a $230 \mathrm{kV}$. Estão incluídos dados de transformadores sem e com contato entre o tanque e o comutador sob carga, embora este último tipo seja em número reduzido. A intenção do uso deste conjunto neste formato é para estabelecer uma estimativa qualitativa da generalização possível entre grupos de características heterogêneas. O conjunto é dividido em dois tipos de falhas conforme sua origem, ou seja, térmica ou elétrica e a condição de normalidade. Assim ocorre um agrupamento das características relativas a descargas elétricas e faixas de temperatura em dois grupos. Neste caso constam informações quanto à potência nominal do transformador, tempo de operação, tipo de cobertura (ar/conservador), tensões de trabalho e tipo de comutador. Os dois conjuntos de dados estão nos anexos de (Zirbes, 2003).

Para o estabelecimento de referenciais de desempenho é normalmente utilizada a relação entre o número de casos com diagnóstico correto e o número total de casos. Para o estabelecimento destes referenciais, foram aplicadas algumas das metodologias de diagnóstico apresentadas nos conjuntos de dados citados acima. Como as diferentes metodologias apresentam diversas formas de caracterização dos diagnósticos, para uma uniformização das formas de identificação de falhas foi adotada a nomenclatura da IEC. Para a equalização das descrições foram observadas as possíveis causas de falhas relatadas e associadas com a classificação adotada. $\mathrm{Na}$ tabela 11 são caracterizados os métodos aplicados, estabelecendo uma identificação para uso posterior nas demais tabelas e os valores globais de acerto dos diagnósticos para dois grupos.

Os critérios aplicados para caracterização da correção dos diagnósticos foram diferentes para os dois grupos. No caso dos dados da IEC TC10 foram considerados os diagnósticos conforme sua própria identificação, isto é, segregando por tipo de origem da falha e por intensidade. Uma exceção neste caso é o método de Doernenburg, onde o número de diagnósticos é muito reduzido, portanto não sendo comparável aos demais neste caso. No caso dos dados do CEPEL foram consideradas para efeito de diagnóstico apenas as origens das falhas, não considerando a intensidade das mesmas. Isto porque, nem todos os casos estão claramente identificados. Este procedimento poderia alterar os resultados comparativos entre os diferentes grupos, havendo uma tendência a um melhor desempenho no segundo caso. Entretanto se observa que a maioria dos métodos apresenta uma redução de sua eficiência, no caso dos dados do CEPEL. 
Tabela 11: Dados gerais de diagnósticos obtidos nos conjuntos de dados IEC e CEPEL

\begin{tabular}{|c|c|c|c|}
\hline \multicolumn{2}{|c|}{$\begin{array}{c}\text { Métodos de } \\
\text { diagnóstico }\end{array}$} & \multicolumn{2}{c|}{$\begin{array}{c}\text { Diagnósticos } \\
\text { corretoss (\%) }\end{array}$} \\
\hline Item & $\begin{array}{c}\text { Descrição do método } \\
\text { M1 }\end{array}$ & $\begin{array}{c}\text { Dados } \\
\text { IEC } \\
\text { TC10 }\end{array}$ & $\begin{array}{c}\text { Dados } \\
\text { CEPEL }\end{array}$ \\
\hline M21 & $\begin{array}{c}\text { Mótodo de } \\
\text { original }\end{array}$ & 65.1 & 55.7 \\
\hline previsto na IEEE \\
C57.104-91 \\
M6
\end{tabular}

${ }^{1}$ Foram considerados como condição normal os casos onde o método não é aplicável

${ }^{2}$ Foi considerado apenas o limite superior dos valores sugeridos como condição normal

Para uma avaliação dos resultados apresentados é necessário observar, além do acima citado, a característica do método e a composição dos conjuntos de dados. Alguns dos métodos, embora apresentem critérios de identificação da condição de normalidade a partir das concentrações de gases ou de suas relações, utilizam como critério para a validação de sua aplicação a taxa de formação dos gases. Este critério de certa forma atua como um identificador da condição de normalidade. No caso dos dados disponíveis não é possível sua utilização uma vez que as taxas são desconhecidas. Assim, o desempenho destes métodos pode ser influenciado pela condição de normalidade na composição relativa dos conjuntos de dados. Por outro lado, a condição de normalidade se sobrepõe à condição de falha térmica de baixa intensidade. Os resultados mostram diferenças entre os conjuntos de dados. Outro aspecto que se verifica é que o método de Rogers apresenta melhor desempenho na identificação de falhas do que na identificação da normalidade.

Para uma melhor avaliação dos aspectos acima citados são apresentados os resultados para os grupos de transformadores e dos reatores no caso dos dados da IEC TC10. No caso dos transformadores os casos de normalidade são significativos, sendo inexistentes no caso de reatores. Da mesma forma a distribuição das falhas térmicas é diferenciada. No caso dos dados do CEPEL a condição de normalidade é mais significativa, embora ocorra um maior equilíbrio entre os casos.

A Tabela 12 apresenta estes dados segregados. É possível constatar que os vários métodos apresentam uma variabilidade significativa na sua eficiência conforme a origem e composição dos conjuntos de dados a serem diagnosticados.

\section{MÉTODO DE DIAGNÓSTICO BASEADO EM REDES NEURAIS}

A rede neural do tipo perceptron de multi-camadas (MLP) é freqüentemente utilizada para a função de classificação. Com raras exceções, os trabalhos publicados, com aplicações de redes neurais em sistemas de diagnóstico da condição de equipamentos elétricos, utilizam este tipo de rede para seu desenvolvimento. É um tipo de rede relativamente simples

Tabela 12: Dados de diagnósticos corretos por conjunto de dados

\begin{tabular}{|c|c|c|c|c|c|}
\hline Método & \multicolumn{2}{|c|}{$\begin{array}{c}\text { Dados IEC - } \\
\text { diagnósticos } \\
\text { corretos (\%) }\end{array}$} & \multicolumn{3}{c|}{$\begin{array}{c}\text { Dados CEPEL - } \\
\text { diagnósticos } \\
\text { corretos (\%) }\end{array}$} \\
\hline Item & $\begin{array}{c}\text { Transfor- } \\
\text { mador }\end{array}$ & Reator & Normal & $\begin{array}{c}\text { Falha } \\
\text { Tér- } \\
\text { mica }\end{array}$ & $\begin{array}{c}\text { Falha } \\
\text { Elé- } \\
\text { trica }\end{array}$ \\
\hline M1 $^{2}$ & 61.1 & 74.1 & 84.3 & 52.5 & 39.3 \\
\hline M2 $^{2}$ & 68.5 & 74.1 & 91.5 & 51.2 & 39.3 \\
\hline M3 & 35.2 & 61.3 & 3.6 & 66.2 & 55.7 \\
\hline M4 & 40.7 & 54.8 & 2.4 & 51.2 & 50.8 \\
\hline M5 & 38.9 & 64.5 & 6.0 & 50.0 & 34.4 \\
\hline M6 & 35.2 & 67.7 & 6.0 & 30.0 & 57.3 \\
\hline M7 & 51.8 & 81.2 & 72.2 & 65.0 & 39.3 \\
\hline
\end{tabular}

${ }^{1}$ Foi considerado apenas o limite superior dos valores sugeridos como condição normal

${ }^{2}$ Foram considerados como condição normal os casos onde o método não é aplicável 
que pode apresentar uma não-linearidade acentuada na formação de planos que definem as áreas dos grupos no espaço multidimensional.

A rede tipo MLP possui uma camada de entrada, uma ou mais camadas intermediárias e uma camada de saída. Cada neurônio é associado a um vetor de pesos, um multiplicador dos vetores, um somador que soma o produto vetorial a uma constante (bias). Assim, o vetor de entrada é multiplicado pelo vetor de pesos e somado a uma constante. Este produto é aplicado a uma função de transferência, normalmente uma função tipo sigmóide. As camadas intermediárias são obtidas a partir da conexão de uma saída de um neurônio da camada anterior à entrada de neurônios que compõem esta camada. O número de componentes do vetor de entrada, o número de saídas, as camadas intermediárias e a forma de interconexão das mesmas definem normalmente as características da arquitetura da rede. Um outro aspecto a ser considerado na arquitetura é a utilização de uma única rede com múltiplas saídas ou múltiplas redes com uma saída e a sua forma de conexão. O treinamento deste tipo de rede, que normalmente envolve um tempo maior que outros tipos de redes, é realizado apresentando os dados de entrada e verificando os resultados que a rede fornece. Estes resultados são comparados com as saídas esperadas para aqueles dados de entrada e calculado o erro quadrático médio. A correção dos pesos é feita da saída para a entrada (backpropagation) utilizando por exemplo o método do gradiente para minimização do erro.

A utilização de uma arquitetura de rede onde os dados relativos à concentração ou relação de concentração de gases de um caso representam o vetor de entrada da mesma e o uso de múltiplas saídas para identificação dos diagnósticos simultaneamente não apresentou bons resultados, a exemplo do relatado em casos publicados na literatura. A utilização de múltiplas redes, com idênticos dados de entrada e com uma única saída identificando uma dada condição foi a que apresentou melhores resultados. O diagnóstico é definido, por um algoritmo, a partir das saídas individuais das redes de forma hierárquica. Desta forma, é verificada inicialmente a condição de normalidade ou falha, caso seja identificada a condição como sendo de falha, é identificada sua origem, se térmica ou elétrica. Após esta identificação é verificada a condição de sua intensidade, ou seja, se a descarga elétrica é caracterizada como de baixa ou alta intensidade ou o sobreaquecimento. Esta estrutura pode ser visualizada na Figura 2.

As redes foram treinadas de forma que as saídas correspondentes a cada possível diagnóstico tenham valor unitário ou zero, e a caracterização da condição dada por um valor acima de 0.7 ou abaixo de 0.3. Desta forma, valores intermediários são caracterizados como "sem diagnóstico". Foi utilizada a função sigmóide com valores entre zero e um. Outros valores de saída associados a outras funções de ativação e outras faixas de variação não apresentaram resultados significativos.

Cada uma das redes individualmente processa os mesmos dados de entrada e apresenta na saída a classificação correspondente ao seu treinamento. A classificação de estado é feita de forma hierárquica seguindo a seqüência lógica de diagnóstico. $\mathrm{O}$ algoritmo da rede foi implementado utilizando o Matlab e com processamento sequiencial, onde cada rede é processada em função dos resultados anteriores.

Foram verificadas também, outras formas de grupamento de identificação das condições, como, por exemplo, entre a condição de normalidade e a falha térmica e entre a falha de origem elétrica. Os resultados não foram significativos, como poderia se esperar, em função da sobreposição destas condições.

Para o treinamento das redes sempre foi utilizado o grupo de dados de transformadores da IEC TC10, sendo verificada a necessidade de utilização camadas intermediárias.

Uma das dificuldades na aplicação é a forma de representação dos dados de entrada das redes. O uso das concentrações de gases como apresentadas nas análises não permite um ajuste das mesmas dentro de uma faixa de erro razoável. Na literatura normalmente é recomendada a normalização dos dados, neste caso os resultados não foram significativos. O uso das relações de concentrações e posterior normalização apresentou resultados mais significativos.

Os resultados obtidos com uma rede treinada com nove relações de gases são apresentados na Tabela 13. Nos resultados obtidos $67 \%$ dos diagnósticos incorretos no caso dos reatores são devidos à dificuldade de identificação das descargas de baixa intensidade em relação às de alta intensidade. Esta dificuldade também foi observada em outras metodologias. No caso de falhas de origem térmica $82 \%$ dos diagnósticos incorretos são devidos à dificuldade de discriminação entre a condição normal e a falha térmica. É possível observar a diferença de desempenho da rede em função dos grupos onde foi aplicada. As características deste tipo de rede e a intensidade do treinamento podem ser responsáveis por este comportamento. Por outro lado, as diferenças entre os grupos de dados terão o mesmo efeito, na medida em que as regiões definidas em um grupo não encontram correspondência no outro.

Com a mesma arquitetura de rede foi efetuado o treinamento baseado nos casos do grupo de dados do CEPEL. Os resultados do treinamento mostraram uma grande dificuldade de discriminação entre a condição de normalidade e a falha de origem térmica, isto é, a identificação da falha térmica praticamente não ocorreu, enquanto a identificação da condição de normalidade e a da falha de origem elétrica foram ele- 


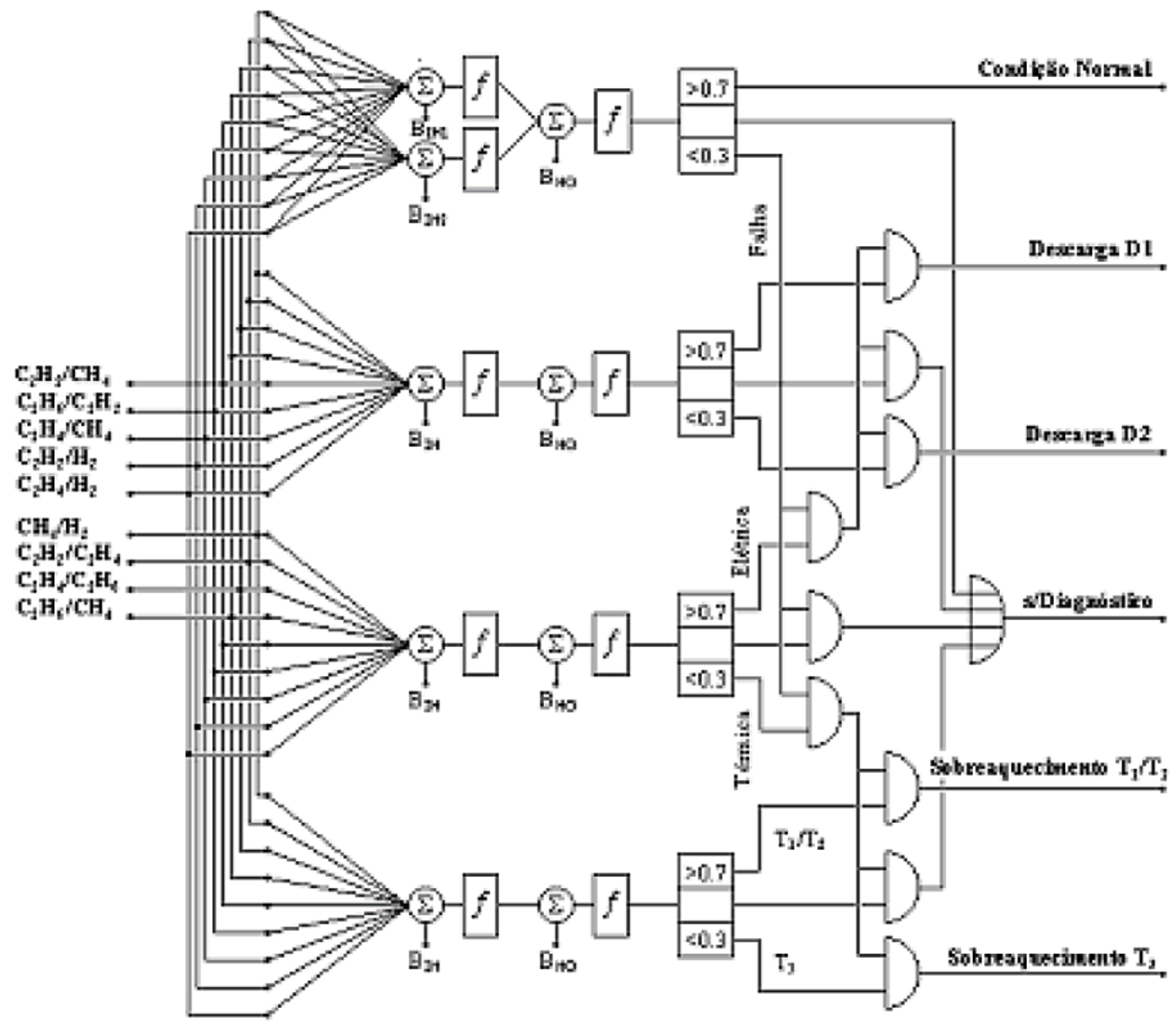

Figura 2: Diagrama esquemático da rede neural múltipla tipo MLP para diagnóstico a partir das relações de concentração de gases.

vadas, superiores a 90\%. Quando esta rede foi aplicada ao conjunto de dados dos transformadores e reatores os resultados foram modestos. Este fato pode ser um indicativo da diferença entre os grupos.

Tabela 13: Resultados de diagnóstico obtidos com uma rede neural tipo MLP, com cinco opções de estado

\begin{tabular}{|c|c|c|c|c|}
\hline \multicolumn{2}{|c|}{$\begin{array}{c}\text { Dados IEC- } \\
\text { diagnósticos } \\
\text { corretos (\%) }\end{array}$} & \multicolumn{3}{|c|}{$\begin{array}{c}\text { Dados CEPEL - } \\
\text { diagnósticos } \\
\text { corretos (\%) }\end{array}$} \\
\hline $\begin{array}{c}\text { Transfor- } \\
\text { mador }\end{array}$ & Reator & Normal & $\begin{array}{c}\text { Falha } \\
\text { Térmica }\end{array}$ & $\begin{array}{c}\text { Falha } \\
\text { Elétrica }\end{array}$ \\
\hline 98.1 & 80.6 & 51.8 & 45.0 & 50.8 \\
\hline
\end{tabular}

O treinamento deste tipo de metodologia é normalmente realizado sendo separado do grupo total uma parte dos dados uma parcela dos mesmos para teste do ajuste e avaliação de erro. Algumas vezes quando o número de componentes do grupo é pequeno são permutados os elementos do grupo de treinamento e do grupo de teste, com isto é possível um melhor controle sobre a capacidade de generalização da rede. Este procedimento não foi aqui adotado.

É interessante observar, independente da aplicação aqui descrita, que a metodologia de treinamento utilizada nesta técnica é muito dependente da representatividade do grupo de dados utilizado em seu treinamento e avaliação. Assim mesmo com um bom desempenho da rede para o grupo utilizado no treinamento e teste, se o mesmo não for representativo do conjunto que se propõe representar, a generalização não é possível. Embora de uma maneira geral todos os métodos apresentem esta dificuldade, este particularmente apresenta uma sensibilidade acentuada neste aspecto. 


\section{CONCLUSÕES}

Conforme pode ser observado na descrição dos itens anteriores, a aplicação dos métodos convencionais envolve uma incerteza significativa. Embora a análise de gases dissolvidos no óleo seja uma das técnicas mais bem sucedidas na determinação de falhas incipientes, é considerada mais como uma arte do que uma ciência. Isto ocorre em função das aproximações e simplificações utilizadas e na ausência de critérios universais. Assim uma análise mais detalhada normalmente é elaborada por um especialista que com sua experiência agrega uma série de informações quanto aos métodos, incertezas e principalmente quanto ao comportamento dos equipamentos específicos envolvidos.

Na ultima década a pesquisa e utilização de técnicas de inteligência artificial aplicadas à análise de gases dissolvidos no óleo têm evoluído significativamente. Vários fatores contribuíram para este fato, a evolução e domínio de aplicação destas técnicas a outros campos de diagnóstico, a facilidade de integração de várias informações e dados, inclusive de diferentes origens, para o auxílio à decisão, o aumento do volume de informações em função do número de equipamentos supervisionados e da importância e reflexos de uma tomada de decisão quanto ao estado de um equipamento.

Os sistemas desenvolvidos não têm por objetivo a substituição do papel exercido por especialistas, mas basicamente servirem de apoio aos mesmos na tomada de decisão. Isto ocorre pela redução do número de informações a serem analisadas, seja pela determinação da severidade ou priorização da análise seja pela apresentação de informações préelaboradas. A utilização de monitoração em linha, por exemplo, implica na adoção de mecanismos de pré-análise para redução do volume de informações, alguns sistemas de análise estão sendo desenvolvidos para processamento em tempo real.

\section{REFERÊNCIAS}

Bozzini C., Davies I., Schliessing H., Schober J., Serena E., Viale F., Wilputte R. (1975). Detection of and research for the characteristics of an incipient fault from analysis of dissolved gases in the oil of an insulation, Electra, $\mathrm{N}^{\circ} 42$.

Doernenburg E., Gerber O.E. (1967). Analysis of dissolved and free gases for monitoring performance of oil-filled transformers, Brown Boveri Review, 54.

Duval M. (1989). Dissolved gas analysis: It can save your transformer, IEEE Electrical Insulation Magazine, Vol. 5, $\mathrm{N}^{\circ} 6$, Novembro/Dezembro.
Duval M., de Pablo A. (2001). Interpretation Of gas-in-oil analysis using IEC publication 60599 and IEC TC 10 databases, IEEE Electrical Insulation Magazine, Vol. $17, \mathrm{~N}^{\circ} 2$, Março/Abril.

Du Y., Mamishev A.V., Lesieutre B. C., Zahn M., Kang S.H. (2001). Moisture Solubility for differently conditioned transformer oils, IEEE Transactions on Dielectrics and Electrical Insulation, Vol. 8, $\mathrm{N}^{\circ} 5$, Outubro.

Galand J., Thibault M., Viale F., Samat J., Vuarchex P. (1972). Application de la chromatographie en phase gazeuse au diagnostic des degradations dans l'ísolation des materials électriques, $R G E$, Vol. 81, $\mathrm{N}^{o} 11$, Novembro.

IEEE Std C57.104-1991 (1991) IEEE Guide for the Interpretation of Gases Generated in Oil-Immersed Transformers".

IEC 60599 (1999). Mineral oil-impregnated electrical equipment in service - Guide to the interpretation of dissolved and free gases analysis, International Electrotechnical Commission.

Wilson, A. C.M. (1980). Insulating liquids: their uses, manufacture and properties, Institution of Electrical Engineers, Peter Pereginus Ltd..

Rouse T.O (1998). Mineral Insulating Oil in Transformers, IEE Electrical Insulation Magazine, Vol. 14, $\mathrm{N}^{o} 3$, Maio/Junho.

Oommen T. V. (1991), On line moisture sensing in transformer, Electrical Electronics Insulation Conference, 1991. Boston ,Outubro.

Kan H., Miyamoto T. (1995). Proposals for an improvement in transformer diagnosis using dissolve gas analysis (DGA), IEEE Electrical Insulation Magazine, Vol. 11, $\mathrm{N}^{o}$ 6, Novembro-Dezembro.

Koritsky Y. (1970). Electrical Enginnering Materials, Mir Publishers.

Milasch, M. (1984). Manutenção de Transformadores em Líquido Isolante, Editora Edgard Blücher LTDA 1984.

Pugh D. R., (1973). Combustible gas analysis, Minutes of Forty International Annual Conference of Double Clients, seção 10-401.

Sokolov V., Bulganova V., Berier Z. (2001). Assessment of power transformer insulation condition, Electrical Insulation Conference and Electrical Manufacturing \& Coil Winding Conference. 
Sokolov V., Berier Z., Rashkes V. (1999). Effective methods of assessment of insulation system conditions in power transformers: a view based on practical experience, Electrical Insulation Conference and Electrical Manufacturing \& Coil Winding Conference.

Rogers R.R. (1975). U.K. Experiences in the interpretation of incipient faults in power transformers by dissolved gasin-oil chromatography analysis (A Progress Report), Minutes of Forty-Second International Conference of Double Clients,seção 10-201;

Projeto NBR 7274 (1999). Interpretação da Análise dos Gases de Transformadores em Serviço, projeto de revisão da NBR 7274/ 1982, Junho.

Zirbes, R. (2003). Metodologias para Avaliação e Diagnóstico do Estado de Isolamentos de Papel Impregnado com Óleo Mineral. Dissertação de Mestrado, Programa de Pós-Graduação em Engenharia Elétrica, Universidade Federal de Santa Catarina. 